\title{
Laser Ablation of Carbon Fiber Reinforced Plastics: Laser-Ionization TOF Mass Spectrometric Study
}

\author{
Aiko NARAZAKI ${ }^{* 1,2}$, Tadatake SATO ${ }^{* 1,2}$, Yoshizo KAWAGUCHI ${ }^{* 1,2}$ and Hiroyuki NIINO ${ }^{* 1,2}$ \\ ${ }^{* 1}$ Research Institute for Innovation in Sustainable Chemistry, National Institute of Advanced Indus- \\ trial Science and Technology (AIST), Central 5, 1-1-1 Higashi, Tsukuba, Ibaraki 305-8565, Japan \\ E-mail: narazaki-aiko@aist.go.jp \\ *2 Advanced Laser and Process Technology Research Association (ALPROT), Japan
}

\begin{abstract}
Neutral fragments from a nanosecond $\mathrm{KrF}$ excimer laser-ablated carbon fiber reinforced plastic (CFRP, carbon fiber/epoxy resin composite) were investigated by laser-ionization time-of-flight (TOF) mass spectrometry in order to explore the laser ablation dynamics of CFRP. The neutrals were detected after the post-ionization by an $\mathrm{ArF}$ excimer laser pulse at variable delay times, $\mathrm{t}_{\mathrm{d}}$, relative to the ablation $\mathrm{KrF}$ excimer laser pulse. Depending on the ablation fluence, TOF mass spectra and laser-etched surface morphology drastically changed. At the laser fluence of $0.3 \mathrm{~J} \cdot \mathrm{cm}^{-2}$, the resins were preferentially ablated. The TOF mass spectra contained a main peak at $36 \mathrm{amu}$ assigned to $\mathrm{C}_{3}{ }^{+}$in early delay times, and then the main peak changed to $39 \mathrm{amu}$ assigned to $\mathrm{C}_{3} \mathrm{H}_{3}{ }^{+}$. At 1.0 and $1.5 \mathrm{~J} \cdot \mathrm{cm}^{-2}$, both of carbon fibers and resins were ablated. The most prominent peak was $\mathrm{C}_{3}{ }^{+}$independent of the delay time. The delay-time distribution of $\mathrm{C}_{3}{ }^{+}$exhibited a single peak at $0.3 \mathrm{~J} \cdot \mathrm{cm}^{-2}$ and then changed to two peaks at $1.5 \mathrm{~J} \cdot \mathrm{cm}^{-2}$. The fits with shifted Maxwell-Boltzmann distribution gave good agreement, suggesting transient maximum CFRP temperature of 4700, 6400, and $7100 \mathrm{~K}$ at $0.3,1.0$ and $1.5 \mathrm{~J} \cdot \mathrm{cm}^{-2}$, respectively.
\end{abstract}

DOI: $10.2961 / \mathrm{jlmn} .2014 .01 .0012$

Keywords: CFRP, KrF excimer laser ablation, neutral fragment, TOF mass spectrometry, laser ionization

\section{Introduction}

Carbon fiber reinforced plastic (CFRP) is a composite material with both higher strength derived from carbon fibers and lighter weight compared to many metals. Therefore, CFRP has become one of major structural materials applicable to automotive, aerospace and marine industries, and so forth. Although the mechanical drilling of CFRP is still common practice in industry, it often causes serious material damage such as delamination, fiber pull out and inadequate surface roughness of the cut walls. Tool wear is another problem with the mechanical drilling due to abrasive nature of the carbon fibers. Laser as a no-contact and fast tool can be used for cutting of CFRP [1-5]. However, the mixture of fibers and polymers in CFRP remains heterogeneous since each of the components retains their individual physical properties. This needs to optimize laser irradiation conditions carefully for high-quality cutting of both parts of carbon fibers and plastics with different physical properties.

Time-of-Flight (TOF) mass spectrometry gives us the masses and kinetic energies of laser-ablated neutral fragments, which are useful for better understanding of laser ablation dynamics [6-10]. Particularly laser-ionization TOF mass spectrometry can detect laser-ablated neutral species which are post-ionized through a UV laser pulse irradiation with high photon energy such as an ArF excimer laser pulse.

In this work, we have investigated nanosecond $\mathrm{KrF}$ excimer laser ablation plume of CFRP by laser-ionization TOF mass spectrometry in order to explore UV laser abla- tion dynamics of CFRP. The KrF excimer laser was chosen as an ablation light source because UV lasers have been used to minimize laser-induced heat-affected zones (HAZ) compared with other nanosecond pulsed lasers at longer wavelength $[11,12]$.

\section{Experimental}

A CFRP plate with a PAN-type fiber content of $50 \%$ and epoxy resin matrix (from Toray Industries, Inc.) was used as a sample. Laser ablation of the CFRP was performed by a $\mathrm{KrF}$ excimer laser (Compex110, Lambda Physik; $\lambda=248 \mathrm{~nm}$, full width at half-maximum (FWHM) $\approx 20 \mathrm{~ns}$ ) in a vacuum chamber, which was evacuated to a base pressure of $<5 \times 10^{-5} \mathrm{~Pa}$. The repetition rate was kept at $1 \mathrm{~Hz}$ to avoid any cumulative effect. The laser light was directed to the target at an incident angle of around $45^{\circ}$ to the surface normal with a spot size of $0.2 \mathrm{~mm}^{2}$. Through an attenuator, the ablation fluence was set at $0.3,1.0$ and 1.5 $\mathrm{J} \cdot \mathrm{cm}^{-2}$.

Figure 1 shows a schematic diagram of a reflectiontype TOF mass spectrometer (D850, R.M. Jordan) arranged for probing neutrals. The CFRP target was rotated at $1 \mathrm{rpm}$ during the TOF mass measurements to avoid formation of a deep hole. Neutral fragments passing through a repeller applied with a high positive voltage were ionized between the repeller and extraction grids by an $\mathrm{ArF}$ excimer laser pulse (Compex102, Lambda Physik; $\lambda=193$ nm, FWHM $\approx$ $20 \mathrm{~ns}$ ) at variable delay times, $t_{\mathrm{d}}$, relative to the ablation pulse. To minimize the fragmentation, the ArF excimer laser pulse for photoionization was set at relatively low 
fluence. The voltages on the repeller grid, retarding grid and reflection plate were adjusted to reflect the ions toward a microchannel plate (MCP). The time delay of the ArF excimer laser pulse from the ablation laser pulse was controlled with a delay generator (DG-535, Stanford Research). The detected signals were accumulated 30 times on a digital oscilloscope (9310AL, LeCroy) to improve a signal-to-noise ratio. We also carried out the laser-ionization TOF mass spectrometry of highly oriented pyrolytic graphite (HOPG) plate (Digital Instruments, HOPG grade-ZYH) as a reference carbon material.

The surface morphology of the laser-ablated CFRP samples was observed with a confocal scanning laser microscope (Keyence, VK-8500). For the observation, 1000, 500 and 500 shots were irradiated at the same position of the CFRP sample without rotation in vacuum at $0.3,1.0$ and $1.5 \mathrm{~J} \cdot \mathrm{cm}^{-2}$, respectively.

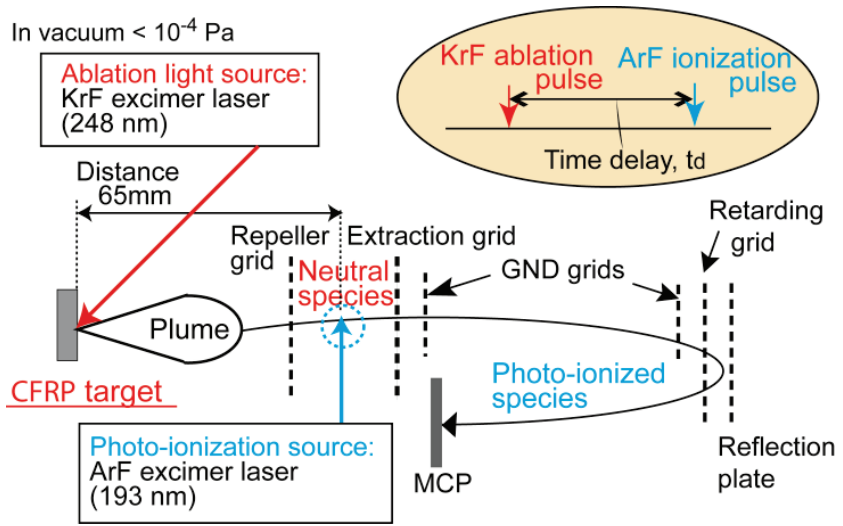

Fig. 1. Schematic of laser-ionization TOF mass apparatus.

\section{Results and discussion}

\subsection{Surface morphology of laser-ablated CFRP}

Figure 2 shows confocal scanning laser microscopic images of CFRP laser-irradiated at (a) 0.3, (b) 1.0 and (c) $1.5 \mathrm{~J} \cdot \mathrm{cm}^{-2}$, respectively. While the surface layer composed of epoxy resins was ablated, no obvious damage of carbon fibers was confirmed in Fig. 2 (a). This is because vaporization temperature of carbon fibers is much higher than those of polymers $[3,13]$. At the higher laser fluences, the fiber-etched area appeared, where both the carbon fibers and resins were obviously laser-etched. It is noteworthy that there was a resin-removed region surrounding the fibber-etched area in Figs. 2 (b) and (c).

Generally, when the laser ablation wavelength is shorter, the penetration depth of the laser light in CFRP becomes smaller. This shallow laser penetration results in ablation with less heating of the bulk material, decreasing heat damages such as formation of a heat affected zone (HAZ). Another advantage of UV laser ablation is the high photon energy which enables the bond scission by photochemical process, carrying out smooth ablated surfaces and clean edges $[14,15]$. The photochemical and photo-thermal nature of the bond scission has been argued especially in UV
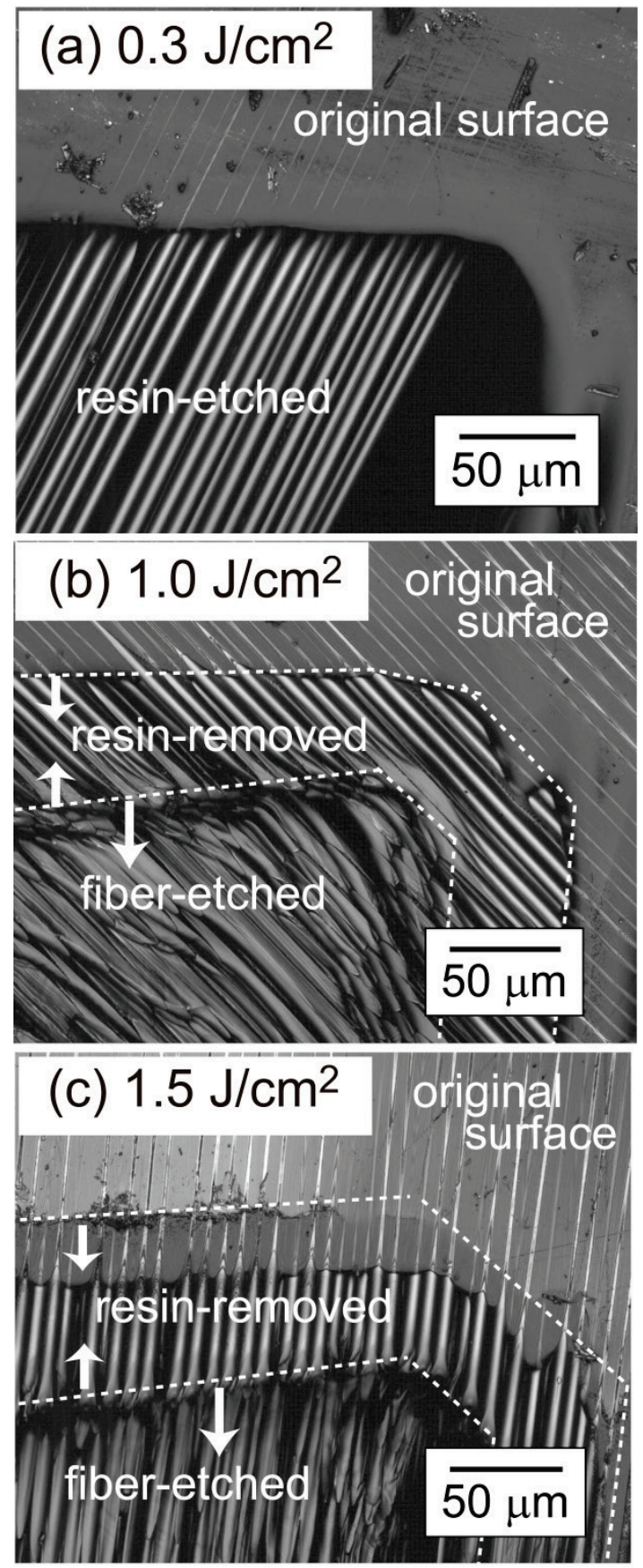

Fig.2 Confocal scanning laser microscopic images of CFRP laser-etched at the laser fluences of (a) 0.3 , (b) 1.0 and (c) 1.5 $\mathrm{J} \cdot \mathrm{cm}^{-2}$. The number of shots were 1000,500 and 500 for 0.3 , 1.0 and $1.5 \mathrm{~J} \cdot \mathrm{cm}^{-2}$, respectively.

laser ablation processes. Sato et al. reviewed laser ablation of polymers [16] and pointed out that the photochemical / photo-thermal nature of the process mainly depends on the identity of materials and experimental conditions such as laser fluence, wavelength, and pulse duration. Srinivasan et al. reported that both photochemical and photo-thermal mechanisms contribute in the etching of polyimide (PI) and polymethylmethacrylate (PMMA) at 193 and $248 \mathrm{~nm}$ [17]. At low fluences, thermal effects were negligible. At higher fluences, thermal effects became significant. Hansen and Robitaille reported that arrival time measurements on the 
$248 \mathrm{~nm}$ ablation of polycarbonate (PC) were well described by Maxwell-Boltzmann velocity distributions, suggesting that the photo-thermal process is dominant even at $248 \mathrm{~nm}$ [18].

For CFRP, it was reported that the excimer laser machining was effective for reducing a HAZ to 5-30 $\mu \mathrm{m}$ compared with longer wavelength $[11,12]$. This suggests that the shallow laser penetration due to UV light absorption by both resins and carbon fibers leads to the ablation of CFRP without the large formation of HAZ. However, there still remained a small HAZ, suggesting the possibility that the laser ablation of CFRP accompanied heat generation to some extent even at UV wavelength.

\subsection{Laser fluence dependence of TOF mass spectra}

Figure 3 shows TOF mass spectra for the laser ablation of the CFRP at $0.3 \mathrm{~J} \cdot \mathrm{cm}^{-2}$ at five post-ionization delay times, $t_{\mathrm{d}}$ of 20, 40, 50, 60 and $90 \mu \mathrm{s}$. The nature of peaks and their intensity changed, dependent on the delay time. In the range of $t_{\mathrm{d}}$ earlier than $60 \mu \mathrm{s}$, the main peak with the highest intensity was at $36 \mathrm{amu}$ and corresponds to the $\mathrm{C}_{3}{ }^{+}$. Other peaks observed at 12, 37 and 39 amu were assigned to $\mathrm{C}^{+}, \mathrm{C}_{3} \mathrm{H}^{+}$and $\mathrm{C}_{3} \mathrm{H}_{3}{ }^{+}$. With an increase in $t_{\mathrm{d}}$, some peaks appeared at 41, 64, 75, 87, 116 and 127 amu. Because these high-mass fragments were not assigned to $\mathrm{C}_{n}{ }^{+}$, they can be considered as a direct fragment from the epoxy part of the CFRP. After $t_{\mathrm{d}}=60 \mu \mathrm{s}$, the highest peak changed from $\mathrm{C}_{3}{ }^{+}$ to $\mathrm{C}_{3} \mathrm{H}_{3}{ }^{+}$. Since only the surface layer composed of epoxy resins was etched after the laser irradiation with 1000 shots as shown in Fig. 2 (a), the detected fragments including small carbon clusters of $\mathrm{C}_{\mathrm{n}}^{+}(\mathrm{n}=1-3)$ in Fig. 3 might mainly come from the epoxy part of CFRP.

Figure 4 (a) shows TOF mass spectra of the CFRP sample laser-ablated at $1.0 \mathrm{~J} \cdot \mathrm{cm}^{-2}$ at post-ionization delay times of 8, 10, 20, 40 and $60 \mu \mathrm{s}$. Independent of the delay time, the most prominent peak was observed at $36 \mathrm{amu}$ assigned to $\mathrm{C}_{3}^{+}$and peaks assigned to $\mathrm{C}^{+}$and $\mathrm{C}_{2}^{+}$appeared at 12 and $24 \mathrm{amu}$. This tendency is similar with TOF mass spectra obtained for HOPG as a reference material. In addition, both carbon-fibers and epoxy resins were clearly etched as shown in Fig. 2 (b). From these facts, the laser ablation of carbon fibers occurred at $1.0 \mathrm{~J} \cdot \mathrm{cm}^{-2}$ and the major fragments of $\mathrm{C}_{3}{ }^{+}$came from both of fibers and resins. Figure 4 (b) shows a TOF mass spectrum at a delay time of $40 \mu \mathrm{s}$ at $1.0 \mathrm{~J} \cdot \mathrm{cm}^{-2}$. The inset is an enlarged spectrum in a high-mass region at a magnification of approximately 40 times. There were some small peaks in a high-

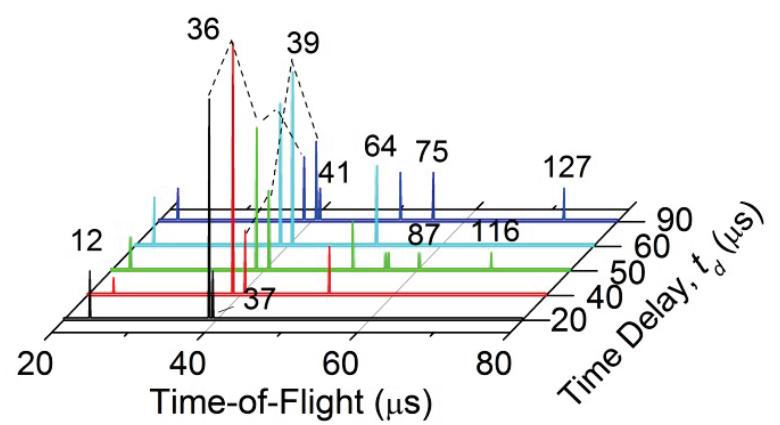

Fig.3 Series of TOF mass spectra obtained from the CFRP laser-ablated at a fluence of $0.3 \mathrm{~J} \cdot \mathrm{cm}^{-2}$. (a)
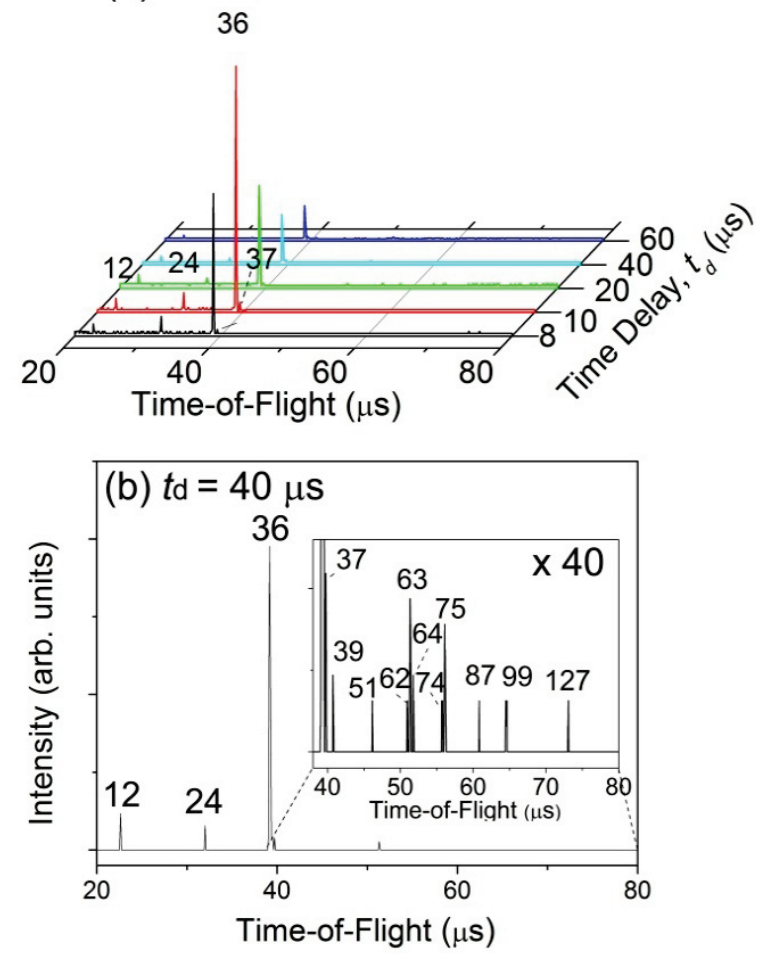

Fig.4 (a) Series of TOF mass spectra obtained from the CFRP ablated at $1.0 \mathrm{~J} \cdot \mathrm{cm}^{-2}$. (b) TOF mass spectrum at $t_{\mathrm{d}}=40 \mu \mathrm{s}$. In addition to peaks at $m / q=12,24,36$, many weak peaks were observable in a high mass range.

mass region, which were not assigned to $\mathrm{C}_{n}^{+}$. These species might stem from the dissociation of the resin polymer, similar with the high-mass species observed at $0.3 \mathrm{~J} \cdot \mathrm{cm}^{-2}$.

At $1.5 \mathrm{~J} \cdot \mathrm{cm}^{-2}$, a typical TOF mass spectrum with a postionization delay time of $11 \mu \mathrm{s}$ is shown in Fig. 5. The most prominent peak appeared at $36 \mathrm{amu}$ assigned to $\mathrm{C}_{3}{ }^{+}$and peaks assigned to $\mathrm{C}^{+}, \mathrm{C}_{2}^{+}$and $\mathrm{C}_{3} \mathrm{H}^{+}$were also observed at 12, 24 and $37 \mathrm{amu}$. This behavior is similar with the previous TOF mass spectra at $1.0 \mathrm{~J} \cdot \mathrm{cm}^{-2}$ and these peaks came from both of the carbon fibers and resins.

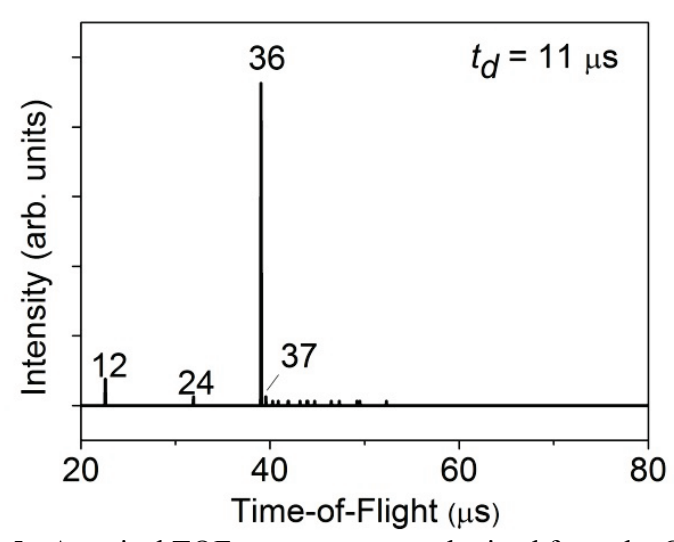

Fig.5 A typical TOF mass spectrum obtained from the CFRP ablated at a fluence of $1.5 \mathrm{~J} \cdot \mathrm{cm}^{-2}$. 


\subsection{Delay-time distribution of $\mathrm{C}_{3}^{+}$species}

In order to explore the laser fluence effect on plume dynamics of CFRP, delay-time distribution of the major $\mathrm{C}_{3}{ }^{+}$was investigated at different fluences. In our previous report [10], it was found that a shifted Maxwell-Boltzmann (SMB) distribution gave better fitting than a MaxwellBoltzmann (MB) distribution in the case of $\mathrm{C}_{3}{ }^{+}$fragments produced by the $\mathrm{KrF}$ laser ablation of CFRP. Thus, we conducted fitting of experimental data with a following SMB equation:
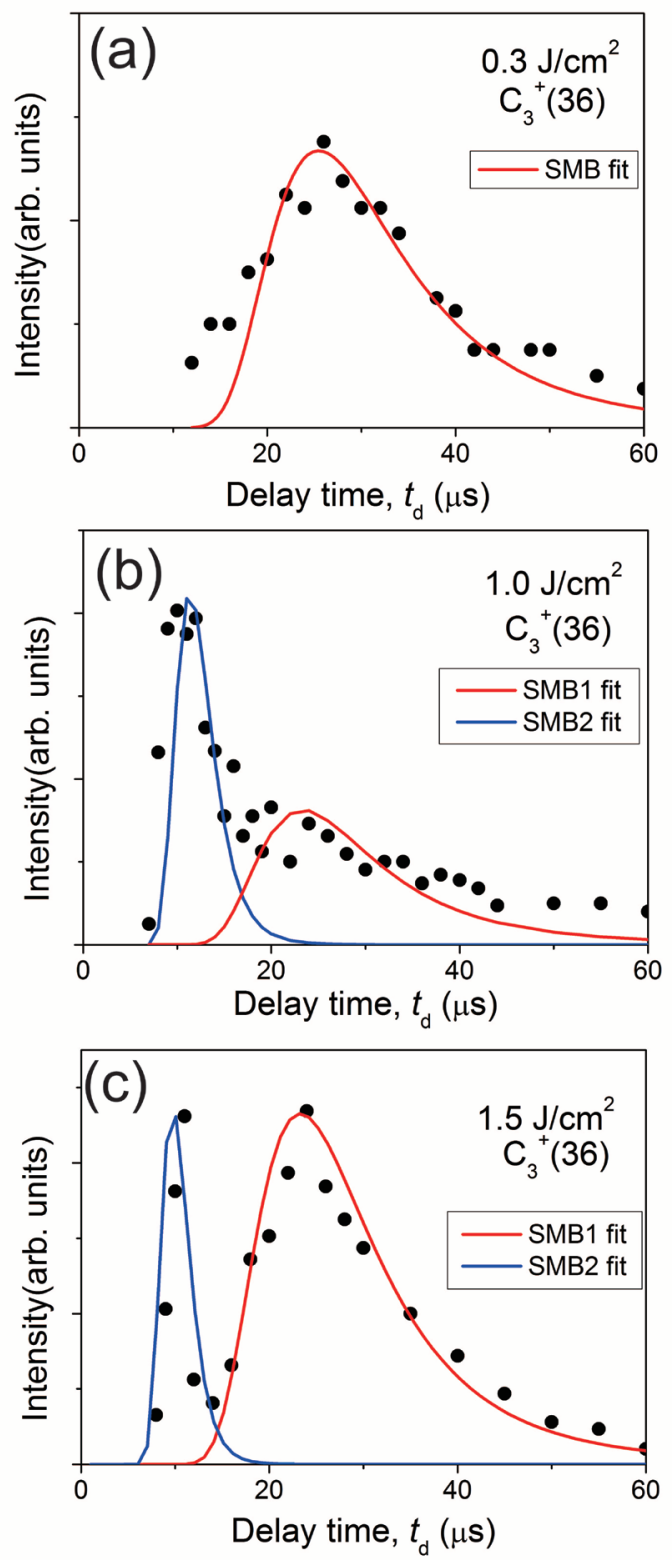

Fig.6 (a) Delay-time distribution of $\mathrm{C}_{3}{ }^{+}$at different laser fluences of (a) 0.3 , (b) 1.0 and (c) $1.5 \mathrm{~J} \cdot \mathrm{cm}^{-2}$. (a) The red curve stands for a fit of $\mathrm{C}_{3}{ }^{+}$to SMB distribution with $\mathrm{COM}$ velocity of $1.2 \times 10^{3} \mathrm{~m} \cdot \mathrm{s}^{-1}$ and KL temperature of $3300 \mathrm{~K}$. (b) The red and blue curves stand for fits by SMB distributions with COM velocity of $1.7 \times 10^{3} \mathrm{~m} \cdot \mathrm{s}^{-1}, T_{\mathrm{K}}$ of $3300 \mathrm{~K}$ and $5.0 \times 10^{3} \mathrm{~m} \cdot \mathrm{s}^{-1}$, $4500 \mathrm{~K}$, respectively. (c) The red and blue curves denote fits by SMB distributions with COM velocity of $1.7 \times 10^{3} \mathrm{~m} \cdot \mathrm{s}^{-1}, T_{\mathrm{K}}$ of $3300 \mathrm{~K}$ and $6.0 \times 10^{3} \mathrm{~m} \cdot \mathrm{s}^{-1}, 5000 \mathrm{~K}$, respectively.

$$
f(\mathrm{t})=A^{\prime} t^{-4} \exp \left[-\left(m / 2 k_{B} T_{K}\right)\left(L / t-v_{g}\right)^{2}\right]
$$

where $A^{\prime}$ is an amplitude scaling factor, $m$ stands for the mass of the fragment, $k_{\mathrm{B}}$ is the $\mathrm{MB}$ constant, and $L$ corresponds to the distance between target and the photoionization point. $T_{K}$ stands for the Knudsen layer (KL) temperature and $v_{g}$ is the center-of-mass (COM) velocity. If collisions among the fragments become prominent, the Knudsen layer formation adequately describes the ablation plume dynamics, which extents up to a few mean free paths from the target surface and where such collisions frequently occur. As a result, the flight distribution of the fragments changes from a MB to SMB. Therefore, the better agreement with the SMB distribution indicates the existence of frequent collisions among the fragments in the CFRP ablation plume in this work.

Figure 6 shows the delay-time distribution of major $\mathrm{C}_{3}{ }^{+}$ fragments from laser ablation of CFRP at (a) 0.3, (b) 1.0 and (c) $1.5 \mathrm{~J} \cdot \mathrm{cm}^{-2}$, respectively. The red and blue curves stand for the SMB distributions obtained by fitting the experimental data (solid circles).

At $0.3 \mathrm{~J} \cdot \mathrm{cm}^{-2}$, there was a single peak. The red curve is a fit to this peak by SMB distribution with COM velocity of 1.7 $\times 10^{3} \mathrm{~m} \cdot \mathrm{s}^{-1}$ and $T_{\mathrm{K}}$ of $3300 \mathrm{~K}$.

With an increase in laser fluence, the delay-time distributions changed from a single peak in Fig. 6 (a) to a broad peak with a tail in Fig. 6 (b) and obvious two peaks in Fig. 6 (c). In the case of $1.0 \mathrm{~J} \cdot \mathrm{cm}^{-2}$, this kind of broad peak could not be fitted well with any single MB or SMB distribution. Thus, we tried to fit the data with two SMB peaks. Consequently, the combination of two SMB distributions (the blue and red curves) gives good agreement with the experimental data, as shown in Fig. 6 (b). The red solid curve is a fit to the tail part of the broad peak by SMB distribution with COM velocity of $1.7 \times 10^{3} \mathrm{~m} \cdot \mathrm{s}^{-1}$ and $T_{\mathrm{K}}$ of $3300 \mathrm{~K}$ The blue curve shows a fit of the faster peak to SMB distribution with COM velocity of $5.0 \times 10^{3} \mathrm{~m} \cdot \mathrm{s}^{-1}$ and $T_{\mathrm{K}}$ of $4500 \mathrm{~K}$

In Fig. 6 (c) at a fluence of $1.5 \mathrm{~J} \cdot \mathrm{cm}^{-2}$, the red and blue curves of the SMB distributions with COM velocity of 1.7 $\times 10^{3} \mathrm{~m} \cdot \mathrm{s}^{-1}, T_{\mathrm{K}}$ of $3300 \mathrm{~K}$ and $6.0 \times 10^{3} \mathrm{~m} \cdot \mathrm{s}^{-1}, 5000 \mathrm{~K}$ are good fits for slower and faster components. The kinetic energies of the slower and faster $\mathrm{C}_{3}{ }^{+}$were calculated to be 0.54 and $6.8 \mathrm{eV}$.

Kokai and Koga reported the time-of flight mass spectrometry for UV laser ablation of graphite $(\lambda=266 \mathrm{~nm}$, pulse width $\approx 8 \mathrm{~ns}, 1 \mathrm{~Hz}$ ) [7]. The major neutral species were $\mathrm{C}_{3}{ }^{+}$. The $\mathrm{C}_{3}{ }^{+}$arrival time distributions were fitted well with $\mathrm{MB}$ and $\mathrm{SMB}$ distributions at 0.4 and $0.6 \mathrm{~J} \cdot \mathrm{cm}^{-2}$, respectively. At $0.6 \mathrm{~J} \cdot \mathrm{cm}^{-2}$, KL temperature was obtained to be $4250 \mathrm{~K}$ comparable with those obtained in this work. Furthermore, it is noteworthy that laser ablation of graphite at both 266 and $1064 \mathrm{~nm}$ gave the same major species of $\mathrm{C}_{3}$ [19], which were similar with pyrolysis of graphite by other methods [20].

In our previous report [21], we have investigated plume dynamics of ns UV laser ablation $(\lambda=355 \mathrm{~nm}$, pulse width $\approx 8 \mathrm{~ns}, 5 \mathrm{~J} \cdot \mathrm{cm}^{-2}$, in air) of the CFRP sample employed in this work by time-resolved imaging and spectroscopy of plume emission. Initially the plume emission spectra consisted of sharp lines from excited ablated species. Then the 
spectra changed to a broad emission from blackbody radiation with several thousands of $\mathrm{K}$.

Although the photo-chemical ablation process can contribute to the UV laser ablation of CFRP as pointed out above, the photo-thermal one is considered to play a major role in our work because the delay-time distributions of major $\mathrm{C}_{3}^{+}$species were reasonably well described by shifted Maxwell-Boltzmann distributions over the entire fluence range in addition to the observation of $\operatorname{HAZ}[11,12]$. Thus, transient surface temperature of CFRP was estimated from the KL temperature under a photo-thermal assumption. Under the assumption of thermally-driven process, the KL temperature might be about $30 \%$ lower than the target surface temperature [22]. Thus-obtained transient maximum CFRP temperatures were 4700,6400 , and $7100 \mathrm{~K}$ at 0.3 , 1.0 and $1.5 \mathrm{~J} \cdot \mathrm{cm}^{-2}$, respectively.

In general, typical delay-time distribution of laserablated fragments is composed of a single peak for various compounds such as polymers, graphite and other materials like silicide [6-9]. However, the two peaks were observed in Fig. 6 (c). One of possible explanations is as follows. The CFRP contains two kinds of components of carbon fibers and resins with much different vaporization / decomposition temperatures. Thus, each peak corresponds to the fragment emitted from each of the components. Further, the intensity of the slower peak increased with increasing the laser fluence from 1.0 to $1.5 \mathrm{~J} \cdot \mathrm{cm}^{-2}$. It is possible that this increase of the slower fragments come from the resinremoved area in Fig. 2 where a polymer component seemed to evaporate preferentially at higher fluence, although it needs further exploration. Anyway, the main $\mathrm{C}_{3}{ }^{+}$fragments were emitted from both of carbon fibers and resins at higher fluence. The transient temperature of laser-irradiated CFRP surface drastically increased with an increase in laser fluence, resulting in a maximum temperature of $7100 \mathrm{~K}$ at $1.5 \mathrm{~J} \cdot \mathrm{cm}^{-2}$.

\section{Conclusion}

We have investigated neutral fragments emitted from nanosecond $\mathrm{KrF}$ excimer laser ablation of a CFRP (carbon fiber/epoxy resin composite) using laser-ionization TOF mass spectrometry. Neutral fragments from the laser ablation of CFRP were ionized by an ArF excimer laser pulse at variable delay times, $t_{\mathrm{d}}$, relative to the ablation pulse. The surface morphology of laser-ablated area drastically changed with an increase in the laser fluence. While only the resins were etched at $0.3 \mathrm{~J} \cdot \mathrm{cm}^{-2}$, both of the carbon fibers and resins were ablated at 1.0 and $1.5 \mathrm{~J} \cdot \mathrm{cm}^{-2}$. TOF mass spectra varied dependent on the laser fluence. At 0.3 $\mathrm{J} \cdot \mathrm{cm}^{-2}$, the main peak was at $36 \mathrm{amu}$ assigned to $\mathrm{C}_{3}{ }^{+}$in early delay times, and then the main peak changed to 39 amu assigned to $\mathrm{C}_{3} \mathrm{H}_{3}{ }^{+}$. These fragments came from the resin part. At higher fluence than $1 \mathrm{~J} \cdot \mathrm{cm}^{-2}$, the most prominent peak was $\mathrm{C}_{3}{ }^{+}$, independent of the post-ionization delay time. From the fitting of delay-time distribution of major $\mathrm{C}_{3}{ }^{+}$species with a shifted Maxwell-Boltzmann distribution, transient maximum temperature of CFRP was estimated to be 4700,6400 and $7100 \mathrm{~K}$ at $0.3,1.0$ and $1.5 \mathrm{~J} \cdot \mathrm{cm}^{-2}$, respectively.

\section{Acknowledgments}

This work was supported in part by the national project of "Advanced Laser and Processing Technology for Nextgeneration Materials Project” (High-power Pulsed Fiber Laser and Processing Technology Project) which was launched in 2010FY (period: 5 years) in Industrial Technology Center of NEDO.

\section{References}

[1] P. Moreno, C. Méndez, A. García, I. Arias and L. Roso: Appl. Surf. Sci., 252, (2006) 4110.

[2] D. Herzog, P. Jaeschke, O. Meier and H. Haferkamp: International Journal of Machine Tools \& Manufacture, 48, (2008) 1464.

[3] Z. L. Li, H. Y. Zheng, G. C. Lim, P. L. Chu and P. L. Li: Composites Part a-Applied Science and Manufacturing, 41 (2010) 1403.

[4] C. Emmelmann, M. Petersen, A. Goeke, and M. Canisius: Physics Procedia, 12, (2011) 565.

[5] H. Niino and R. Kurosaki: Proc. of SPIE, 7920, (2011) 792019

[6] F. Kokai, Y. Koga, Y. Kakudate, M. Kawaguchi, S. Fujiwara, M. Kubota and K. Fukuda: Appl. Phys. A, 59, (1994) 299.

[7] F. Kokai and Y. Koga: Nucl. Instrum. Methods B, 121, (1997) 387.

[8] C. Grivas, H. Niino and A. Yabe: Appl. Phys. A, 69, (1999) S159.

[9] A. Narazaki, T. Sato, Y. Kawaguchi and H. Niino: Appl. Surf. Sci., 208, (2003) 52.

[10]A. Narazaki, T. Sato, Y. Kawaguchi, R. Kurosaki and H. Niino: Proc. Int. Symp. on Laser Processing for CFRP and Composite Materials 2013, Yokohama, (2013) p.4-1

[11]H.K. Toenshoff, F. von Alvensleben, P.B. Heekenjann and G. Willmann: Proc. of the SPIE-The International Society for Optical Engineering, (1997) 108.

[12]F. Voelkermeyer, R. Kling and B. Denkena: Proc. CFK-Valley Stade Convention, (2007) p.135.

[13] W. R. Creasy: Polymer, 33, (1992) 4486.

[14] S. Kreling, F. Fischer, R. Delmdahl, F. Gäbler and K. Dilger: Physics Procedia, 41 (2013) 282.

[15] F. Fischer, S. Kreling and K. Dilger: Physics Procedia, 39 (2012) 154.

[16] H. Sato and H. Nishio: J. Photochem. Photobiol. C: Photochemistry Reviews, 2 (2001) 139.

[17] R. Srinivasan, M. A. Smrtic and S. V. Babu: J. Appl. Phys., 59 (1986) 3861.

[18] S. G. Hansen and T. E. Robitaille: J. Appl. Phys., 64 (1988) 2122.

[19]F. Kokai: T. IEE Japan, 117-C, (1997) 1194.

[20]D. J. Krajnovich: J. Chem. Phys., 102, (1995) 726.

[21] Y. Kawaguchi, T. Sato, A. Narazaki, R. Kurosaki and H. Niino: Proc. Int. Symp. on Laser Processing for CFRP and Composite Materials 2013, Yokohama, (2013) p.4-2.

[22] R. Kelly and R. W. Dreyfus: Surf. Sci., 198, (1988) 263.

(Received: July 18, 2013, Accepted: February 3, 2014) 